\title{
TELEDERMATOLOGY: CONCEPTS AND APPLICATIONS IN MEDICINE
}

\author{
Maria-Magdalena Constantin ${ }^{1,2}$, Anca Răducan ${ }^{1}$, Elena Poenaru' ${ }^{2}$, Traian Constantin ${ }^{2,3}$ \\ ${ }^{1}$ IInd Department of Dermatology, Colentina Clinical Hospital, Bucharest, Romania \\ ${ }^{2}$, ,Carol Davila" University of Medicine and Pharmacy, Bucharest, Romania \\ ${ }^{3}$ Urology Department, ,, Prof. Dr. Th. Burghele“ Clinical Hospital, Bucharest, Romania
}

\begin{abstract}
Objectives. To assess the concepts of teledermatology (TD), a subspecialty of dermatology and one of the most common applications of telemedicine.

Material and methods. This systematic review, totaling 32 works, is aimed to evaluate the advantages and limitations of teledermatology, which can be delivered in two ways: real-time (synchronous), using videoconference technology, and store-and-forward (SAF) methods (aynchronous), using digital images via internet, a cheaper and more convenient modality for the health care provider.

Results. So far, teledermatology has been implemented in dermoscopy, dermatopathology and chronic wound care. However, other chronic skin disease, such as psoriasis, dermatitis, acne, rosacea and skin cancers can be evaluated and monitored using store-and-forward methods.

Conclusions. In order to develop a teledermatology platform, Romanian dermatology requires specific protocols and equipment for taking and sending photographs. A dermatologist would then review the data using store-andforward method and respond with a diagnosis and therapeutic algorithm.
\end{abstract}

Keywords: teledermatology, store-and-forward, chronic leg ulcers, psoriasis

Telemedicine is a worldwide practice, providing remote specialty healthcare, using telecommunication technologies such as audio, video and data communication to evaluate, diagnose and treat patients over a distance. Throughout history, people have tried different ways of long distance communication, especially in terms of healthcare, because a doctor's medical opinion was essential and also hard to get. The roots of ,in absentia care“ or distance medicine date back to ancient times, when Galen (129-200 A.D.), who was considered very skilled, sometimes used to prescribe to patients without ever seeing them, offering consultations by letter, for which he in fact received generous rewards. (1) Also, african villagers used smoke signals to warn others of potential deadly disease or epidemics. Hundreds of years later, it was Willem Einthoven, a Dutch physician, who first transferred electrocardiograms at long distance in 1905, followed by the development of radio consultations in the 1920s from medical centers for patients aboard ships at sea and on remote islands in Norway, Italy, and France; then, in the early 1950s transmission of radiographic images in the United States has emerged. History shows that when in need for medical support, people will find a way, maybe an ingenious one to get in touch with a doctor. (2)

\section{INTRODUCTION TO TELEDERMATOLOGY}

Teledermatology (TD) is a subspecialty in the medical field of dermatology and is one of the most common applications of telemedicine and e-health. Due to its predominant visual nature, dermatology seems to better benefit from telemedicine, probably more than any other specialties (3) In fact, teledermatology was developed in 1995 by the dermatologists Perednia and Brown as an application of telemedicine, described in a scientific publication as a cost effective way of providing remote healthcare 
service for people living in rural regions underserved by dermatologists. (4) Since then, it has been noticed an ongrowing interest in this field, especially with the development of dermoscopy. TD is widely used in industrialized nations such as USA, Canada, United Kingdom, Saudi Arabia, France and Norway, which was the first country to reimburse telemedicine since 1996. (5)

Having in mind that the majority of dermatologists work in urban areas, TD allows patients to save both time and money, by eliminating the costs of travel and offering access to otherwise unavailable medical consultation. TD applications include consultation, evaluation, diagnosis, treatment and follow up. TD can be delivered in 2 ways: Realtime/ Live interactive TD (synchronous), using videoconference technology, and Store-and-forward (SAF) methods (aynchronous), using digital images via internet (e-mail, web-platform), a cheaper and more convenient modality for the health care provider. (6-8) Other concepts include hybrid models (which combine SAF and real time models), online discussion groups (ODG), mobile TD (uses smartphone or tablet applications), teledermatopathology, teledermoscopy and telesurgery. $(6,7)$

\section{CONCEPTS OF TELEDERMATOLOGY}

Telemedicine consulting center: in this site the patient is present and the equipment for taking and transferring patient data is available. In these centers, a general practitioner is usually present and he is responsable for communicating and forwarding the patient's data to a specialist. (9)

Telemedicine specialty center: this is the site where the specialist is present. The specialist analyses the data received from the telemedicine consulting center via GP or interacts directly with the patient through a live feed. (9)

Store and Forward Teledermatology: Store and forward (SAF) communication involves sending photographs and the patient data from a general practioner to a specialist for storage and consultation. GP can take good quality images in a short time. $(6,8,10)$ The specialist examines the data such as images and other details of the patient and gives his opinion to the referring physician $(6,7,9)$. The main advantages of the SAF method are inexpensiveness, as it is very cost effective mode in terms of equipment costs, easy to set up and use. Large number of images can be taken and analysed in a short time. (11) Quality of the image may be very good, with the advent of new and performant digi- tal cameras. The quality of the digital camera used is a major factor in determining the accuracy of diagnosis. Increased coverage, the images taken by a GP can be sent to many specialists and furthermore the stored images can be analysed by many specialists. (12)

Real time/Live interactive teledermatology: patients directly interact with the dermatology provider via videoconferencing. The main advantage of this method is that proper medical history can be taken. A great advantage over the SAF method is that in real time TD other regions can also be examined at the same time if necessary, not just the affected area (13). Another benefit of this method is interaction, as the patient is in direct video contact with the specialist and can ask questions directly. Diagnostic accuracy has been confirmed by various feasibility studies when videoconferencing is compared to face-to-face consultations. $(6,7,8)$

Hybrid model: it combines the SAF model and the Real time model, and is very useful as it avoids misunderstandings, saves time and achieves both physician and patient satisfaction. (14)

Mobile Teledermatology: in this method mobile phones, tablets or personal digital assistants (PDA devices) are used to capture and transfer the images. Patients with certain conditions such as leg ulcers can benefit from this method as patients themselves, nurses or GPs can send images periodically to a specialist. With the help of smartphones and high quality digital cameras it has become possible to take good quality images, which can be sent to a specialist via internet directly through mobiles or PDA devices. $(6,7,9)$

Teledermatopathology: in this model histopathological images are transmitted for expert opinion. It is a very useful tool in areas or countries where dermatopathologists or histopathologists are very few in number. $(15,16)$

Teledermoscopy: dermoscopic images can be sent for evaluation and opinion to an expert using SAF method and virtual slide systems (VSS). By digitally scanning an entire glass slide at a high resolution virtual slides are made and the images are sent to a storage system $(6,9)$.

Telesurgery: the concept of teleconsultation has been extended to telesurgery which requires a medical assistant or a local practitioner who has access to the patient and a specialist in another remote location. The specialist guides the local physician through audio and video communication to perform the procedure (9).

Online Discussion groups (ODG): difficult to manage cases represent a great challenge to the 
health care system. With the help of a group of dermatologists an ODG can be formed. Difficult or interesting cases can be discussed by the specialists in these groups $(6,7,8,9)$.

\section{ADVANTAGES OF TELEDERMATOLOGY}

Patients may benefit from a series of advantages that teledermatology brings: access to specialty care, better triage and management service, rapid diagnosis and care, access to second-opinion, screening for skin cancers, avoidance of white coat syndrome, elimination of long waiting list, better education of the patient, earlier detection of complications and therefore less emergency consultations and a decrease in the frequency of hospitalization in patients with chronic wounds, reduced travel costs, comfort for patients.

Even though face-to-face interaction between patient and specialist is the gold standard in dermatology, TD has proven to be a useful tool, acceptable for both patients and dermatologists. Patients like to talk to the specialist, and live interactive TD, using real-time videoconferencing is the preferred to store-and-forward, since it is a better substitute for the traditional consultation, but it requires higher costs $(6,17)$. However, SAF is mostly used in TD, but we shouldn't lose focus of what's important: each lesion is unique, and as dermatologists we need a 3D view of the lesion, we need to see the whole picture and to know the medical history.

As far as second-opinion service is concerned, TD brings together specialists from all around the world, allowing a multidisciplinary approach of a case, and gives patients the chance to receive a second opinion from an expert in a certain filed of dermatology, otherwise impossible to obtain.

Teledermatology also provides educational benefits to primary care providers (general practitioners (GP), GP's and home nurses, as well as dermatologists, both young residents and experienced specialists). Young residents can benefit from videoconferences held by leading experts and can access online discussion forums or groups, but they may also be in touch with their mentors, seeking medical advice when faced with a difficult case (17).

So far, teledermatology has been implemented in dermoscopy, dermatopathology and chronic wound care. However, other chronic skin disease, such as psoriasis, dermatitis, acne and rosacea can be evaluated and monitored using store-and-forward methods. Another useful application of TD is the management of skin cancers in general and particularly of melanoma. Studies have assessed high levels of accuracy in TD diagnosis for melanocytic and non-melanocytic "pink" lesions, compared to traditional face-to-face consultations, supporting once again the reliability of TD in screening for skin cancers (18).

Chronic leg ulcers care involves follow-up, which in many cases is difficult, mainly for elderly patients living in remote areas. Performing TD in such cases requires initial hospitalization or consultation to assess the extent of the wound and to initiate proper treatment. Afterwards, TD can be successfully used, studies showing $71 \%$ improvement of leg ulcers for patients in which follow-up care was performed at home by a nurse, by means of TD (19).

Domoplaies is a French inter-regional project management aiming to improve the quality care for wounds, offering patients a multidisciplinary approach for chronic wounds by using a telemedicine platform, electronic medical records, computers for the dermatologists and mobile tablets with $3 \mathrm{G}$ internet access for the home nurses (20).

Psoriasis patients are also candidates for TD, since the disease is chronic and patients require frequent hospitalizations. TD may increase compliance and could be used in monitoring patients undergoing biologic therapy or phototherapy $(21,22)$. Both children and adults suffering the burden of atopic dermatitis or vitiligo may also benefit from TD (23).

Considering that there is a shortage of pediatric dermatologists, TD can prove useful in facilitating general physicians, pediatricians and dermatologists access to medical opinion of academic or experienced pediatric dermatologists (24).

In 2009 a new acronym has emerged: CAP-HAT encompassing the main factors necessary for TD: dermatology case (C), approach (A), purpose (P), health care specialists involved $(\mathrm{H})$, and type of teledermatology tools (T). Practically, CAP-HAT can be applied in two ways: for spot diagnosis and for follow-up in chronic cases (23).

TD can be helpful when used in rural communities or remote areas (fishing communities, islands, mountains, deserts), poor countries, regions facing natural disasters (floods, hurricanes, earthquakes), war or terrorism areas. In addition, TD has been implemented with good results in nursing homes, prisons, emergency departments, oil platforms, military exercises-Aircraft carriers, forest training, submarines, survival training and spatial stations (25). Studies have shown that TD is useful for institutionalised psychiatric patients with chronic skin diseases (26) and HIV positive patients (27). 
Furthermore, patients are more receptive to SAF-TD if the area that is subjected to consultation involves chest, legs, genitals and face. Young patients are more open to the use of technology in medical care $(6,17,23)$.

Routine SAF method can also be used to screen the conditions and triage the patients suitable to undergo telesurgery (keloids, scars, hemangiomas, laser assisted hair reduction). In time, this method may help increase the skills of the local practitioners or medical assistants, who will be able to perform similar procedures over a wide variety of patients (9).

\section{MAIN CONCERNS AND LIMITATIONS IN TD}

The main challenges in TD include technical issues, such as lack of access to internet, slow internet connection, incompatibility between software and poor quality images, which may lead to diagnostic uncertainty, and therefore a higher demand for biopsies, also increasing the overall costs (28).

One limitation of TD is that in SAF method the specialist is not in direct contact with the patient, thus, sometimes it is difficult to obtain other relevant data which might be required by the specialist and can be time consuming. Also, the patient is not able to ask questions directly to the specialist because of the lack of direct communication between the two. Absence of interaction can lead to lower level of patient satisfaction. Other limitations include the possible discomfort of the patient to be getting examined in front of a camera rather than face-to-face consultation, the lack of availability of trained health care workers at the remote centers and limited or no access to the prescribed medications, which is a great failure to the purpose of TD even if other things are in place $(8,28,29)$.

Another important aspect to consider is that the estimated time for a teledermatology consultation may in fact be longer than in the case of traditional consultation (30 minutes, as opposed to $15 \mathrm{~min}$ utes), because of the challenges in evaluating and treating patients through means of telecomunications $(17,29)$.

The limitation of telesurgery in dermatology is that very complex procedures or the conditions which require direct intervention by the specialist cannot be performed by this method. Besides, technical failure and poor internet connection can lead to interruption of the procedure or surgery. Complications that may arise during the procedure, which are beyond the skills of medical assistant, cannot be attended immediately (9).
Reimbursement for the referring physician and for the dermatologist should be taken into consideration for a better implementation of teledermatology, since the Ministry of Health or the insurance companies do not cover the costs in this field of telemedicine, nor the elaboration of a professional software, available at a national level.

Legal aspects such as licensure laws and malpractice concerns are the downsides of teledermatology, considering the high risk of error in diagnosing and virtually treating patients, lacking face-to-face interactions $(8,28,29)$. One question still needs an answer: should legal responsability be shared by the dermatologist, the GP and the home nurse?

Usually, patients are open to teledermatology, but the main concern is that the confidential personal medical information may be compromised when transmitting data via internet. Patients should be explained what TD implies and informed about who has access to their medical data and photos. Furthermore, a written consent for storing and sending patient's data is compulsory before TD is applied. As a rule, all protective measures should be taken in order to ensure a secure storage and transmission of patient's health information, and patients must be reassured that the confidentiality will not be broken $(30,31)$. Therefore, the medical data hosting server should be authorized by the Romanian authorities, ensuring the data security.

Despite the advantages that TD brings, some physicians, especially dermatologists, are still reluctant to the innovative technology, which they consider impersonal. Due to this controversy, that TD creates a gap between the patient and the dermatologist, a Romanian survey among dermatologists is helpful to establish the potential use of a TD platform in Romania.

\section{ROMANIA AND TELEDERMATOLOGY}

In order to develop a TD platform, Romanian dermatology requires specific protocols and equipment for taking and sending photographs. TD is based on a multidisciplinary approach, between general physician, infectious disease specialist, rheumatologist, pediatrician and diabetologist. According to our proposed protocol, when patients undergo conventional consultation for skin disease, the referring clinician forwards the patient's medical data to the dermatologist, using TD platform service, after receiving the written consent of the patient. The standardized electronic medical record should include the following data: description of the lesion, anatomical location, evolution and dura- 
tion, associated signs and symptoms, personal and family history of skin cancers, history of allergies, childhood dermatitis or asthma, previous treatments for the current condition, other disease.

Sequential digital images should be performed using a digital camera with minimum 8-megapixels resolution and integrated flash. To begin with, a panoramic photo of the body area affected by a certain lesion is necessary, providing a label with the patient's initials, lesion number and date. Next step is taking a close-up photo with scale (placed parallel to the lesion), at a $20 \mathrm{~cm}$ distance from the lesion, using a scale to appreciate the size of the le-

\section{BIBLIOGRAFIE}

1. Susan P.M. The Prince of Medicine: Galen in the Roman Empire. Oxford: Oxford University Press. 2013

2. Ryu S. History of Telemedicine: Evolution, Context, and Transformation. Healthc Inform Res. 2010 March; 16(1): 65-66.

3. Coates S.J., Kvedar J., Granstein R.D. Teledermatology: from historical perspective to emerging techniques of the modern era: part I: History, rationale, and current practice. J Am Acad Dermatol. 2015 Apr; 72(4):563-74.

4. Perednia D.A., Brown N.A. Teledermatology: One application of telemedicine. Bull Med LibrAssoc. 1995; 83:42-7.

5. Hartvigsen G., Johansen M.A., Hasvold P., et al. Challenges in telemedicine and eHealth: lessons learned from 20 years with telemedicine in Tromsø. Stud Health Technol Inform. 2007; 129(Pt 1):82-6.

6. Johnson M.N., Armstrong A.W. Technologies in dermatology: teledermatology review. G Ital Dermatol Venereol. 2011 Apr; 146(2):143-53

7. Krupinski E.A., Antoniotti N., Bernard J. Utilization of the American Telemedicine Association's Clinical Practice Guidelines. Telemed J E Health. 2013 November; 19(11): 846-851.

8. Armstrong A.W., Kwong M.W., Ledo L., et al. Practice Models and Challenges in Teledermatology: A Study of Collective Experiences from Teledermatologists. PLoS One. 2011; 6(12): e28687.

9. Report of the technical working group on telemedicine standardization. Recommended guidelines and standards for practice of telemedicine in India. Technical working group for Telemedicine Standardization Department of Information Technology (DIT), Ministry of Communications and Information Technology (MCIT), Govt. of India. 2003 http://www.sathi.org/Documents/MIT_Standardisation.pdf

10. Berghout R.M., Eminoviæ N., de Keizer N..F, et al. Evaluation of general practitioner's time investment during a store-and-forward teledermatology consultation. Int J Med Inform 2007; 76:S384-91.

11. Wootton R., Blooomer S.E., Corbet R., et al. Multicenter randomized control trial comparing real time teledermatology with conventional outpatient dermatological care: Societal cost benefits analysis. BMJ 2000; 320:1252-6.

12. Loane M.A., Gore H.E., Corbett R., et al. Effect of camera performance on diagnostic accuracy: Preliminary results from the Northern Ireland arms of the UK Multicentre teledermatology trial. J Telemed Telecare 1997; 3:83-8.

13. Baba M., Seçkin D., Kapdaðli S. A comparison of teledermatology using store-and-forward methodology alone, and in combination with Web camera videoconferencing. J Telemed Telecare. 2005; 11:354-60. sion. The last photo (with zoom function) should provide a dermoscopic view of the lesion, connecting the dermatoscope to the digital camera or smartphone/tablet (32).

A dermatologist would then review the data using SAF-TD and respond with a diagnosis and therapeutic algorithm. The referring clinician is responsible for implementing the recommendations and interacting with the patient. As the management plan might require a visit to the dermatologist for further investigations, such as a skin biopsy, the patient should schedule a traditional (face-to-face) consultation to the dermatology clinic.

14. Kaliyadan F., Venkitakrishnan S. Teledermatology: Clinical case profiles and practical issues. Indian J Dermatol Venereol Leprol. 2009; 75:32-5.

15. Weinstein L.J., Epstein J.I., Edlow D., et al. Static image analysis of skin specimens: The application of telepathology to frozen section evaluation. Hum Pathol 1997; 28:30-5.

16. Berman B., Elgart G.W., Burdick A.E. Dermatopathology via a still-image telemedicine system: Diagnostic concordance with direct microscopy. Telemed J 1997; 3:27-32.

17. Massone C., Wurm E.M., Hofmann-Wellenhof R. et al. Teledermatology: an update. Semin Cutan Med Surg. 2008 Mar; 27(1):101-5.

18. Fabbrocini G., Balato A., Rescigno O. Telediagnosis and face-toface diagnosis reliability for melanocytic and non-melanocytic 'pink' lesions. J Eur Acad Dermatol Venereol. 2008 Feb; 22(2):229-34.

19. Binder B., Hofmann-Wellenhof R., Salmhofer W., et al. Teledermatological monitoring of leg ulcers in cooperation with home care nurses. Arch Dermatol. Dec 2007; 143(12):1511-4.

20. http://www.telesante-basse-normandie.fr/l-enrs-et-les-projets/ domoplaies, 1641, 2503.html

21. Koller S., Hofmann-Wellenhof R., Hayn D., et al. Teledermatological monitoring of psoriasis patients on biologic therapy. Acta Derm Venereol. 2011 Oct; 91(6):680-5.

22. Klotz J., Muir L., Cameron C., et al. Monitoring a remote phototherapy unit via telemedicine. J Cutan Med Surg. 2005 Apr; 9(2):47-53.

23. Kanthraj G.R. Patient-assisted teledermatology practice: What is it? When, where, and how it is applied? Indian J Dermatol Venereol Leprol. 2015 Mar-Apr; 81(2):136-43.

24. Philp J.C., Frieden I.J., Cordoro K.M. Pediatric teledermatology consultations: relationship between provided data and diagnosis. Pediatr Dermatol. 2013 Sep-Oct; 30(5):561-7.

25. Miot H.A., Paixão M.P., Wen C.L. Teledermatology: past, present and future. An. Bras. Dermatol. 2005 Oct; 80( 5 ): 523-532.

26. Seghers A.C., Seng K.H., Chio M.T., et al. A prospective study on the use of teledermatology in psychiatric patients with chronic skin diseases. Australas J Dermatol. 2015 Mar 5. doi: 10.1111/ajd.1229

27. Azfar R.S., Weinberg J.L., Cavric G., et al. HIV-positive patients in Botswana state that mobile teledermatology is an acceptable method for receiving dermatology care. J Telemed Telecare 2011; 17:338-40 
28. Moffatt J.J., Eley D.S. Barriers to the up-take of telemedicine in Australia - a view from providers. Rural and Remote Health 2011; 11(2): 1581

29. Desai B., McKoy K., Kovarik C. Overview of international teledermatology. Pan Afr Med J. 2010 Jul 20; 6:3.

30. Stanberry B. The legal and ethical aspects of telemedicine. 1:

Confidentiality and the patient's rights of access. $J$ Telemed Telecare. 1997; 3(4):179-87.
31. Schaffernack I. Legal aspects of teledermatology. J Dtsch Dermatol Ges. 2005 Jul; 3(7):566-8.

32. Piccoli M.F., Amorim B.D.B., Wagner H.M., et al. Teledermatology protocol for screening of Skin Cancer. Anais Brasileiros de Dermatologia. 2015; 90(2):202-210. 